\title{
STUDIES OF ROOM TEMPERATURE ACCELERATOR STRUCTURES FOR THE ILC POSITRON SOURCE*
}

\author{
J. W. Wang, C. Adolphsen, V. Bharadwaj, G. Bowden, V. Dolgashev, R. Jones, \\ E. Jongewaard, J. Lewandoski, Z. Li, R. Miller, SLAC, Menlo Park, CA94025, U.S.A.
}

\section{Abstract}

There are many challenges in the design of the normalconducting portion of the ILC positron injector system such as achieving adequate cooling with the high RF and particle loss heating, and sustaining high accelerator gradients during millisecond-long pulses in a strong magnetic field. The proposed design for the positron injector contains both standing-wave and traveling-wave L-band accelerator structures for high RF efficiency, low cost and ease of fabrication. This paper presents results from several studies including particle energy deposition for both undulator based and conventional positron sources, cooling system design, accelerator structure optimization, RF pulse heating, cavity frequency stabilization, and RF feed system design.

\section{RF STRUCTURES AND PARAMETERS}

Due to the extremely high energy deposition from positrons, electrons, photons and neutrons behind the positron target, the $1.3 \mathrm{GHz}$ pre-accelerator has to use normal conducting structures up to an energy of 250 $\mathrm{MeV}$. A conceptual design of a positron injector for the TESLA linear collider has been proposed and carefully studied. ${ }^{1,2}$ Considering issues of feasibility, reliability and cost savings for the ILC, we've been studying an alternative injector design which consists of both optimized standing wave and traveling wave structures.

\section{SW Structure}

We've designed a simple $\pi$ mode SW structure with 11 cells for the high gradient $(15 \mathrm{MV} / \mathrm{m})$ positron capture structure. The advantages are a more effective cooling system, higher shunt impedance with larger aperture (60 $\mathrm{mm}$ ), lower RF pulse heating, apparent simplicity and cost savings. The mode and amplitude stability for this type of structure has been shown theoretically to be feasible. Figure 1 gives information on the design.
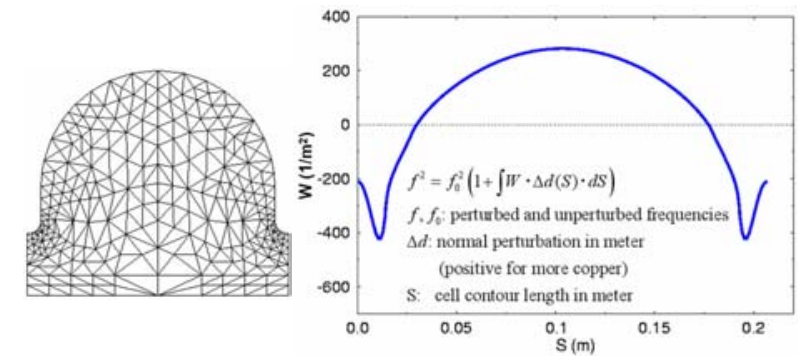

Figure 1: Cell profile of $1.27 \mathrm{~m}$ long $\pi$ mode SW structure and the frequency perturbation weighting function along cell surface.
Table 1: Basic Parameters for SW Structure

\begin{tabular}{|c|c|}
\hline Structure Type & $\pi$ mode \\
\hline Length & $1.27 \mathrm{~m}$ \\
\hline Number of Cells & 11 \\
\hline Aperture (2a) & $60 \mathrm{~mm}$ \\
\hline $\mathrm{Q}_{0}$ & 29700 \\
\hline Shunt Impedance & $34.3 \mathrm{M} \Omega / \mathrm{m}$ \\
\hline $\begin{array}{c}\text { Average Gradient with } \\
\text { 8.6 MW Input Power }\end{array}$ & $15.2 \mathrm{MV} / \mathrm{m}$ \\
\hline
\end{tabular}

\section{TW Structure}

The "phase advance per cell" has been used as a knob for designing large aperture, constant gradient, TW structures with attenuation coefficients $(\tau)$ of $0.5-0.6$ in order to optimize the RF efficiency for different length of TW structures.
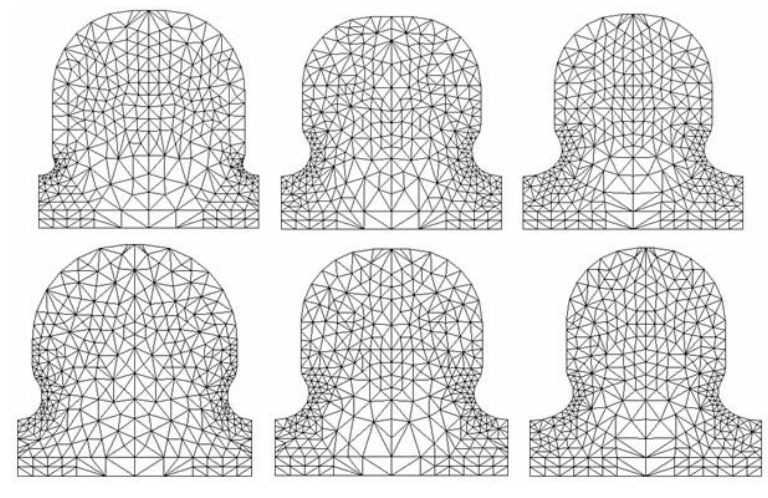

Figure 2: Profiles of the first, middle and last cell for $2.2 \mathrm{~m} 5 \pi / 6$ Mode TW structure (top row) and $4.3 \mathrm{~m} 3 \pi / 4$ Mode TW structure (bottom row).

Table 2: Basic Parameters for TW Structures

\begin{tabular}{|c|c|c|}
\hline Structure Type & $5 \pi / 6$ Mode TW & $3 \pi / 4$ Mode TW \\
\hline Length & $2.2 \mathrm{~m}$ & $4.3 \mathrm{~m}$ \\
\hline Number of Cells & 23 & 50 \\
\hline Aperture (2a) & $46 \mathrm{~mm}$ & $46 \mathrm{~mm}$ \\
\hline Attenuation $\tau$ & 0.55 & 0.61 \\
\hline $\mathrm{Q}_{0}$ & $25870-22300$ & $26510-22760$ \\
\hline $\mathrm{Vg} / \mathrm{c}$ & $0.37 \%-$ & $0.67 \%-$ \\
\hline Shunt & $0.12 \%$ & $0.20 \%$ \\
\hline Impedance & $46.34-36.37$ & $50.05-39.68$ \\
\hline Filling time & $3.2 \mu \mathrm{m}$ & $\mathrm{M} \Omega / \mathrm{m}$ \\
\hline Power Dissipation & $7.8 \mathrm{~kW} / \mathrm{m}$ & $3.6 \mu \mathrm{s}$ \\
\hline Ave. Gradient & $7.7 \mathrm{MV} / \mathrm{m}$ & $8.5 \mathrm{MV} / \mathrm{m}$ \\
at 8.6 MW input & &
\end{tabular}

* Work supported by U.S. Department of Energy, contract DEAC02-76SF00515 
The advantages are lower pulse heating, easy installation for long solenoids, no need for RF reflection protection (circulators), apparent simplicity and cost saving.

\section{FEASIBILITY STUDIES OF SW STRUCTURE FOR POSITRON CAPTURE}

\section{Cooling Design}

Several candidate mechanical designs for the SW cavity cell were considered. The most promising from the standpoint of manufacturability and thermal performance is presented here. The proposed cavity consists of a copper inner body that extends from the cavity iris to the cavity equator. Cooling channels are machined into the outer cylindrical surface and the planar end surface of the copper body (see Figure 3). A copper cover is brazed over the planar end surface and a stainless steel cylinder brazed to the cylindrical outer surface of the copper body. A full cell is formed by brazing two of these half cell assemblies together at the cavity iris and the full accelerator section is assembled from these full cell subassemblies.

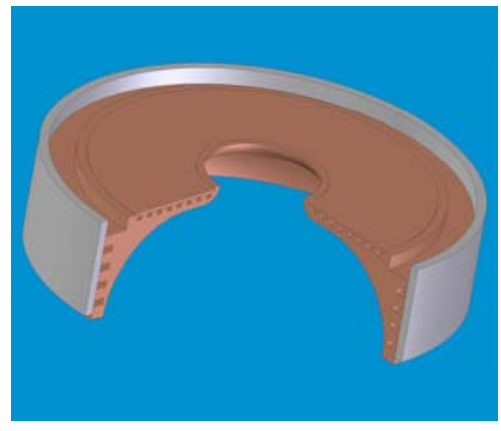

Figure 3: Cutaway view of proposed half cavity.

To determine the operating temperature and the expected tuning changes, an axisymmetric representatio $\mathrm{n}$ was modeled using the general purpose finite element code ANSYS. A two stage analysis was performed where the operating heat loads were applied to a thermal model to determine the temperature field, which in turn was applied as a load to a structural model to determine the thermally induced distortions. Cavity surface RF losses were calculated using the code Omega3P, a finite element parallel eigensolver developed at SLAC. The average volumetric heating $H\left(W / \mathrm{cm}^{3}\right)$ due to particle losses were approximated by fitting an inverse exponential function to particle simulation data ${ }^{4}$ :

$$
H=100 e^{((3-r) / 1.5)}
$$

The particle losses were assumed to be constant along $\mathrm{z}$ and vary only radially (r) as described by equation (1) with $\mathrm{r}$ in $\mathrm{cm}$. Heat transfer coefficients for the cooling channels ranging from $2.7 \mathrm{~W} / \mathrm{cm}^{2 \circ} \mathrm{C}$ for the channels at the cavity outer diameter to $3.0 \mathrm{~W} / \mathrm{cm}^{2 \circ} \mathrm{C}$ for the channels at the cavity iris were calculated assuming a maximum flow velocity of $6.5 \mathrm{~m} / \mathrm{s}$. Several sources suggest that water flow velocities as high as this are acceptable with regard to erosion-corrosion in accelerator applications ${ }^{3}$. The cavity detuning was calculated by applying the Slater perturbation method using the normal cavity surface displacements calculated by ANSYS and weighting factors shown in Figure 1. The results were compared to the cavity frequencies computed using SUPERFISH and found in good agreement.

A series of transient cases using the steady state temperature field as initial conditions were run to determine the temperature excursions during the RF pulse for the conditions of only RF heating and RF heating plus particle heating. The steady state temperature solutions are shown in Figures 4 . The cavity detunings for the steady state and transient cases are summarized in table 3 .

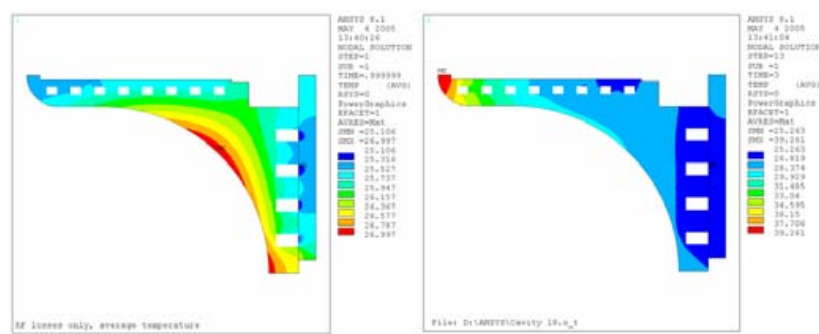

Figure 4: ANSYS thermal model with average RF losses (left) and thermal model with average RF and particle losses (right).

Table 3: Cavity Detunings for Different Loads

\begin{tabular}{|c|c|}
\hline Case & Cavity detuning \\
\hline Average RF losses only & $-20.4 \mathrm{kHz}$ \\
\hline Average RF and particle losses & $-58.6 \mathrm{kHz}$ \\
\hline Start of RF pulse, RF loss only & $-19.5 \mathrm{kHz}$ \\
\hline End of RF pulse, RF loss only & $-23.3 \mathrm{kHz}$ \\
\hline Transient detuning, RF only & $-3.9 \mathrm{kHz}$ \\
\hline $\begin{array}{c}\text { Start of RF pulse, } \\
\text { RF and particle loss }\end{array}$ & $-53.8 \mathrm{kHz}$ \\
\hline $\begin{array}{c}\text { End of RF pulse, } \\
\text { RF and particle loss }\end{array}$ & $-68.9 \mathrm{kHz}$ \\
\hline $\begin{array}{c}\text { Transient detuning, RF and particle } \\
\text { loss }\end{array}$ & $-15.1 \mathrm{kHz}$ \\
\hline
\end{tabular}

\section{Mode Spacing}

The mode spacing near to $\pi$ mode can be expressed as:

$$
\frac{\Delta f}{f} \approx \frac{k \pi^{2}}{4 N^{2}}
$$

For the $1.3 \mathrm{GHz}, \mathrm{N}=11$ cell, $\mathrm{SW}$ structure with coupling coefficient $k=0.0125$, the mode spacing between the working $\pi$ mode and nearest $9 \pi / 10$ mode is $0.4 \mathrm{MHz}$. This is wide enough for stable operation.

\section{Cell Phase Difference}

Due to the energy flow, the cumulative phase shift $\Delta \Phi$ in cell number $n$ from the drive cell can be expressed as:

$$
\Delta \Phi=\frac{\sqrt{1-k}}{k Q_{0}} n^{2}
$$

where $Q_{0}$ is the cell unloaded quality factor.

Figure 5 shows the simulated phase as a function of cell number in the 11-cell SW structure with the input coupler located at the middle. The maximum phase difference $\Delta \Phi$ is $4.7^{\circ}$. The reduction of energy gain due to the phase 
shift summed over 11 cells is $0.18 \%$. The influence of this type of phase difference is negligible for operation.

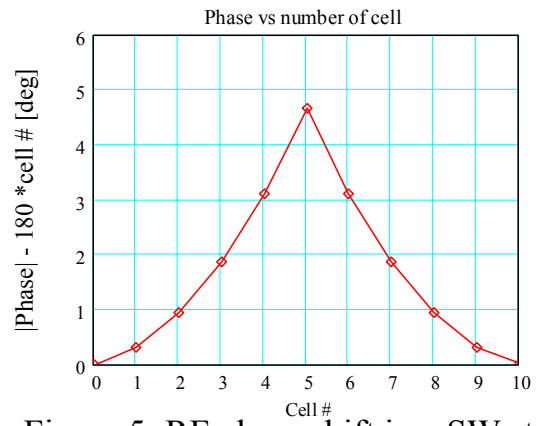

Figure 5: RF phase shift in a SW structure.

\section{RF Amplitude and Phase}

For a high $Q_{0}$ SW structure, the reflection coefficient $\Gamma$ and RF phase $\Phi$ change with the resonant frequency, which drifts with change in average temperature:

$$
\begin{gathered}
\Gamma=-1+\frac{2 \beta}{\beta+1+j \frac{2 Q_{0} \Delta f}{f}} \\
\Phi=\operatorname{Arg}(\Gamma+1)=\tan ^{-1} \frac{2 Q_{0} \Delta f}{f(\beta+1)}
\end{gathered}
$$

For the present design of positron capture section, the transient cavity detuning during $1 \mathrm{~ms}$ RF pulses at maximum particle losses is $15 \mathrm{kHz}$. With optimal cooling water temperature and optimal RF phasing, the acceleration gradient deviation due to power reflection and phase change will be $\sim 2.5 \%$ with no intra-pulse drive phase and amplitude corrections.

\section{RF Amplitude stabilization}

In practical operation, the detuning of each cell can be different due to transient RF filling and uneven particle losses. Figure 6 shows the results from an equivalent circuit simulation for the case of first five cells having frequency changed from $-100 \mathrm{kHz}$ to $+100 \mathrm{kHz}$.
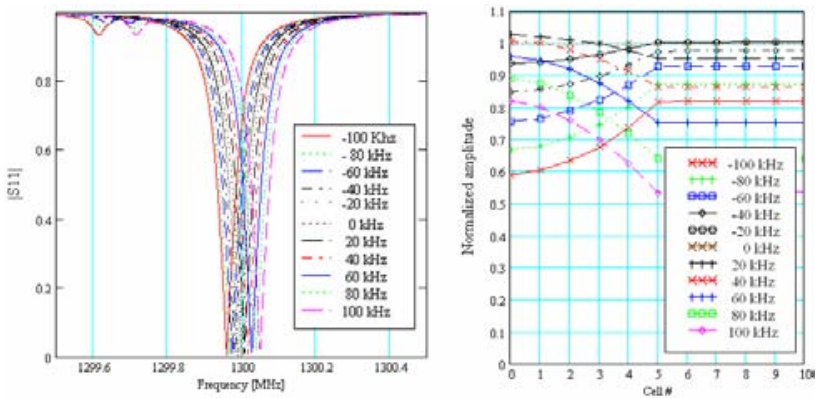

Figure 6: Frequency spectrum (left) and electrical field amplitude (right) for various frequency changes.

It shows that with optimized RF phase adjustment, the SW section can provide acceleration stabilized to better than $2.5 \%$ even for $20 \mathrm{kHz}$ cell frequency detuning.

\section{RF Pulse Heating}

The RF pulse heating $\Delta T$ can be calculated from formula (6), where $H_{t}$ is surface magnetic field, $T_{p}$ is RF pulse width, $\rho$ is the $\mathrm{Cu}$ density, c is its specific heat, $R_{s}$ is its resistivity and $\kappa$ is its thermal conductivity.

$$
\Delta T=H_{t}^{2} \sqrt{T_{p}} \frac{R_{s}}{\sqrt{\pi \rho c \kappa}}
$$

The plot in Figure 7 shows the surface magnetic field profile along the cell. The maximum value of $H_{t}$ for 15 $\mathrm{MV} / \mathrm{m}$ acceleration is $43.8 \mathrm{kA} / \mathrm{m}$. The corresponding temperature rise from RF pulse heating is $8.5^{\circ} \mathrm{C}$. Figure 7 also shows the $3 \mathrm{D}$ coupler surface field. The maximum $H_{t}$ on the coupling iris for $15 \mathrm{MV} / \mathrm{m}$ acceleration is $67 \mathrm{kA} / \mathrm{m}$. The RF pulse heating in this case is $21{ }^{\circ} \mathrm{C}$. These temperature increases are safe for high gradient operation.
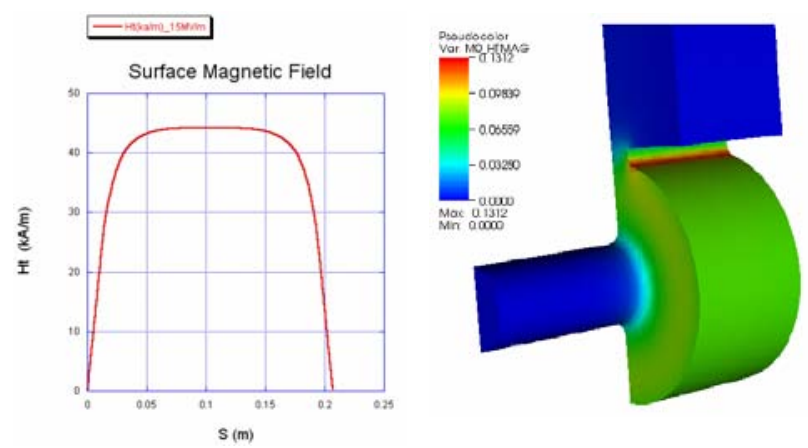

Figure 7: RF pulse heating calculations.

\section{FURURE WORK}

\section{Experimental Studies}

We have designed a 5-cell L-Band test structure with coupler cell at one end and all necessary features for positron capture section. It will be fabricated and high power tested to full gradient and pulse length with an existing power source in the next fiscal year.

\section{Positron Capture Studies}

Capture in structures with initial deceleration has been investigated and experimentally proven to produce a shorter bunch and smaller longitudinal emittance than a conventional capture system ${ }^{5}$. Capture for this approach can be optimized at a lower accelerator gradient. We are also studying this approach for the ILC positron source.

\section{REFERENCES}

[1] K. Flottmann, et al., "Conceptual Design of a Positron Injector for the TESLA Linear Collider, TESLA-0012.

[2] V. Paramonov, "The Cut Disk Structure for High Energy Linacs", PAC1997.

[3] S. Sharma, et al., "An Evaluation of Enhanced Cooling Techniques for High-Heat-Load Absorbers", MEDSI2002 Workshop.

[4] V. Bharadwaj, et al., "Heat Deposition in Positron Sources for ILC, This Conference.

[5] B. Aune and R. Miller, "New Method for Positron Production at SLAC", LINAC1979, SLAC-PUB2393. 